\title{
Rapid Enhancement of Glutamatergic Neurotransmission in Bipolar Depression Following Treatment with Riluzole
}

\author{
Brian P Brennan*, 1,2,3, James I Hudson ${ }^{1,2}$, J Eric Jensen ${ }^{2,4}$, Julie McCarthy ${ }^{5}$, Jacqueline L Roberts', \\ Andrew P Prescot ${ }^{6}$, Bruce M Cohen ${ }^{2,3}$, Harrison G Pope Jr ${ }^{1,2}$, Perry F Renshaw ${ }^{6}$ and Dost Öngür ${ }^{2,3,5}$ \\ 'Biological Psychiatry Laboratory, McLean Hospital, Belmont, MA, USA; ²Department of Psychiatry, Harvard Medical School, Boston, MA, USA; \\ ${ }^{3}$ Shervert Frazier Research Institute, McLean Hospital, Belmont, MA, USA; ${ }^{4}$ Brain Imaging Center, McLean Hospital, Belmont, MA, USA; \\ ${ }^{5}$ Schizophrenia and Bipolar Disorder Program, McLean Hospital, Belmont, MA, USA; ${ }^{6}$ The Brain Institute, University of Utah School of Medicine, \\ Salt Lake City, UT, USA
}

\begin{abstract}
Glutamatergic abnormalities may underlie bipolar disorder (BD). The glutamate-modulating drug riluzole may be efficacious in bipolar depression, but few in vivo studies have examined its effect on glutamatergic neurotransmission. We conducted an exploratory study of the effect of riluzole on brain glutamine/glutamate ( $\mathrm{Gln} / \mathrm{Glu}$ ) ratios and levels of N-acetylaspartate (NAA). We administered open-label riluzole 100-200 mg daily for 6 weeks to 14 patients with bipolar depression and obtained imaging data from 8 - $\mathrm{cm}^{3}$ voxels in the anterior cingulate cortex (ACC) and parieto-occipital cortex (POC) at baseline, day 2, and week 6 of treatment, using two-dimensional J-resolved proton magnetic resonance spectroscopy at $4 \mathrm{~T}$. Imaging data were analyzed using the spectral-fitting package, LCModel; statistical analysis used random effects mixed models. Riluzole significantly reduced Hamilton Depression Rating Scale (HAM-D) scores ( $d=3.4$; $p<0.00 \mathrm{I}$ ). GIn/Glu ratios increased significantly by day 2 of riluzole treatment (Cohen's $d=1.2 ; p=0.023$ ). NAA levels increased significantly from baseline to week $6(d=1.2 ; p=0.035)$. Reduction in HAM-D scores was positively associated with increases in NAA from baseline to week 6 in the ACC $(d=1.4 ; p=0.053)$, but was negatively associated in the POC $(d=9.6 ; p<0.00$ I $)$. Riluzole seems to rapidly increase Gln/Glu ratios — suggesting increased glutamate-glutamine cycling, which may subsequently enhance neuronal plasticity and reduce depressive symptoms. Further investigation of the Gln/Glu ratio as a possible early biomarker of response to glutamate-modulating therapies is warranted.
\end{abstract}

Neuropsychopharmacology (2010) 35, 834-846; doi:I0.1038/npp.2009.191; published online 2 December 2009

Keywords: bipolar disorder; depression; glutamate; N-acetylaspartate; riluzole; MRS

\section{INTRODUCTION}

Bipolar disorder (BD) is a serious mental illness. The manic phase of this disorder is often debilitating, but the depressive phase contributes at least comparable morbidity and mortality (Post, 2005). Although the neurobiology of BD remains incompletely understood, recent evidence has increasingly implicated abnormal glutamatergic neurotransmission (Kugaya and Sanacora, 2005; Öngür et al, 2008; Sanacora et al, 2003). Lacking refined in vivo assays to measure brain glutamate levels, few studies have accurately assessed glutamatergic activity in patients with $\mathrm{BD}$.

Magnetic resonance spectroscopy (MRS) can measure brain glutamate levels in vivo, but signal/noise and spectral resolution problems have forced most studies to quantify glutamate together with glutamine as a composite measure-

*Correspondence: Dr BP Brennan, McLean Hospital, I 5 Mill Street, Belmont, MA 02478, USA, Tel: + | 617-855-29||, Fax: + | 617-8553585, E-mail: bbrennan@partners.org

Received I 4 July 2009; revised 22 October 2009; accepted 26 October 2009 ment labeled 'Glx.' Increased Glx levels have been shown in the depressed (Bhagwagar et al, 2007; Frye et al, 2007), manic (Cecil et al, 2002; Michael et al, 2003), and euthymic (Dager et al, 2004) phases of BD, as well as in rapid-cycling $\mathrm{BD}$ (Michael et al, 2009). However, the physiological significance of Glx is unclear - underscoring the need for separate glutamate and glutamine measures.

Glutamate is continuously recycled between neurons and glia: neuronal glutamate is released into the synapse, taken up by glial cells, and converted to glutamine, which is then shuttled back to neurons and reconverted to glutamate (Rothman et al, 2003). Glutamate levels by themselves do not accurately reflect glutamatergic neurotransmitter activity, because cellular metabolism contributes to the synthesis of glutamate through the tricarboxylic acid (TCA) cycle. Furthermore, static measurements of glutamate and glutamine do not reflect the constant flux though the glutamateglutamine cycle. An aggregate measure, the glutamine/ glutamate (Gln/Glu) ratio, may function as a better (although imperfect) index of glutamatergic neurotransmission than either metabolite alone, because it is more sensitive to changes in the glutamate-glutamine cycle-a 
physiological process that is particularly amenable to this approach, given the reciprocal nature of glutamine and glutamate. As theorized by several other groups, increased Gln/Glu ratios reflect intensified flux through the glutamate-glutamine cycle and an overall increase in glutamatergic neurotransmitter activity (Igarashi et al, 2001; Iltis et al, 2009; Mlynarik et al, 2008; Öngür et al, 2008; Theberge et $a l, 2003,2002)$. It has recently become possible to measure this ratio, using $J$-resolved proton MRS $\left({ }^{1} \mathrm{H}\right.$-MRS), a sequence that acquires spectra at multiple echo times (TEs), allowing improved metabolite quantification (Jensen et al, 2009). Thus, ${ }^{1} \mathrm{H}-\mathrm{MRS}$ can measure glutamate and glutamine levels separately, allowing calculation of Gln/Glu ratios. A recent study using ${ }^{1} \mathrm{H}$-MRS has shown increased Gln/Glu ratios in acutely manic patients, suggesting glutamatergic overactivity in mania (Öngür et al, 2008).

To further investigate glutamatergic activity in $\mathrm{BD}$, one can use ${ }^{1} \mathrm{H}-\mathrm{MRS}$ to assess longitudinal changes in glutamatergic activity after administration of a glutamatemodulating drug probe. This design is especially suited for the depressed phase of the illness, because several antidepressant agents have effects on glutamatergic neurotransmission (Sanacora et al, 2008). Using one of these agents as a drug probe, one could simultaneously analyze changes in both glutamatergic activity and mood symptoms after drug administration. A good candidate for this purpose is riluzole, an FDA-approved medication for the treatment of amyotrophic lateral sclerosis (ALS), which has been shown to potently and rapidly inhibit presynaptic release of glutamate in both in vitro and in vivo studies (Cheramy et al, 1992; Doble, 1996). Riluzole has shown antidepressant effects in preliminary open-label studies of unipolar (Sanacora et al, 2007; Zarate et al, 2004) and bipolar depression (Zarate et al, 2005), and thus represents a promising glutamate modulator/antidepressant probe.

We administered riluzole for 6 weeks to 14 bipolardepressed patients and used $J$-resolved ${ }^{1} \mathrm{H}$-MRS to calculate Gln/Glu ratios at baseline, day 2, and at week 6 of treatment. We obtained ${ }^{1} \mathrm{H}$-MRS data from the anterior cingulate cortex (ACC), a known locus of pathology in BD (Phillips et al, 2003), and from the parieto-occipital cortex (POC), a locus implicated only in unipolar depression (Sanacora et al, 2004), to assess for regional specificity in the biological effects of riluzole. We also analyzed the association between improvement on the 21-item Hamilton Depression Rating Scale (HAM-D) and change in Gln/Glu ratios at day 2 and week 6. We hypothesized that riluzole would improve depression, and that this improvement would be associated with a reduced Gln/Glu ratio in the ACC at day 2 and week 6 . On the basis of previous studies showing an increase in $\mathrm{N}$ acetylaspartate (NAA), a marker of neuronal integrity, after riluzole treatment (Kalra et al, 1998; Mathew et al, 2008), we also hypothesized that riluzole would increase NAA levels in the ACC at week 6 , but not at day 2, given the slow turnover rate of NAA (Choi and Gruetter, 2004).

\section{PATIENTS AND METHODS}

\section{Participant Selection}

Through advertisements, we recruited participants aged 18-65 years, meeting the DSM-IV criteria for BD (type I or II), currently depressed, scoring $\geqslant 18$ on the HAM-D at screening and baseline. Exclusion criteria were as follows: history of schizophrenia or obsessive-compulsive disorder, active psychosis, suicidal risk, alcohol or substance dependence (other than nicotine) within 3 months of enrollment, electroconvulsive therapy within 3 months of enrollment, positive urine drug screen for substances of abuse, previous riluzole treatment, current pregnancy or lactation, history of seizure disorder or organic brain disease, clinically significant medical disease, or MRI contraindication.

During the study, we excluded clozapine, lamotrigine, lithium, carbamazepine, opiates, psychostimulants, atomoxetine, tricyclic antidepressants, and fluvoxamine on the basis of (1) substantial evidence of glutamatergic activity (lamotrigine), (2) previous ${ }^{1} \mathrm{H}-\mathrm{MRS}$ evidence of effects on brain glutamate levels (lithium, opiates, psychostimulants, and atomoxetine), (3) potential effect on riluzole levels (tricyclic antidepressants and fluvoxamine), or (4) risk of neutropenia (clozapine and carbamazepine). Participants taking an excluded medication could enroll after a medication washout of at least five half-lives supervised by the treating psychiatrist or by the study investigator. Participants taking stable doses of nonexcluded medications for $\geqslant 4$ weeks before enrollment were included, provided they required no significant dose changes during the study. No new psychiatric medications were permitted during the study.

\section{Clinical and ${ }^{1} \mathrm{H}$-MRS Evaluation}

Participants received a screening evaluation, at the time of which they signed informed consent as approved by the McLean Hospital Institutional Review Board. We then obtained demographic information, medical/psychiatric history, the Structured Clinical Interview for DSM-IV, physical examination, vital signs, electrocardiogram, and clinical laboratory tests. Thereafter, we administered our primary clinical outcome measure, the HAM-D, and three secondary clinical outcome measures, namely the Montgomery-Asberg Depression Rating scale (MADRS), Clinical Global Impression Scale for Severity (CGI-S), and the Young Mania Rating Scale (YMRS).

Eligible participants returned in $\sim 1$ week for a baseline visit to assess adverse events, concomitant medications, vital signs, HAM-D, MADRS, CGI-S, and YMRS. In addition, participants underwent a ${ }^{1} \mathrm{H}-\mathrm{MRS}$ scan and started a 6-week open-label course of riluzole, $50 \mathrm{mg}$ twice daily (bid) for 14 days, increasing if tolerated to $50 \mathrm{mg}$ in the morning and to $100 \mathrm{mg}$ at bedtime at week 3, and to $100 \mathrm{mg}$ bid at week 4. Participants who were unable to tolerate higher doses could reduce the drug intake to a minimum dose of $50 \mathrm{mg}$ bid. We used riluzole doses over the FDAapproved dose of $100 \mathrm{mg}$ daily on the basis of the mean doses used in previous unipolar (Zarate et al, 2004) and bipolar depression (Zarate et al, 2005) studies.

Participants initiated riluzole on the morning of the day after baseline (day 1). They returned on day 2, after their morning dose, for a second ${ }^{1} \mathrm{H}$-MRS scan, and were assessed for adverse events, concomitant medications, and vital signs, but received no clinical measures. Participants were then seen weekly at weeks 1 through 6 . At each visit, we 
administered the same outcome measures as at baseline, plus the CGI-I (Clinical Global Impression Scale-Improvement). We conducted liver function tests and a complete blood count every 2 weeks, more frequently than the recommended 4 weeks, to closely monitor participants for hepatotoxicity or neutropenia. At week 6, participants received the third ${ }^{1} \mathrm{H}$-MRS scan, and began tapering riluzole by $50 \mathrm{mg}$ per week, with discontinuation after 1 week at $50 \mathrm{mg}$ bid.

\section{MRI/MRS Scans}

Subjects underwent standard T1-weighted structural MRI in a Siemens 3-T Trio scanner (Erlangen, Germany). Those with structural abnormalities as determined by an official radiology report were excluded.

We have previously described the details of MRS acquisition and data analysis (Jensen et al, 2009; Öngür et al, 2008). Briefly, ${ }^{1} \mathrm{H}-\mathrm{MRS}$ acquisitions were conducted on a 4-T scanner (Varian/UnityInova, Varian, Palo Alto, CA, USA), using a volumetric-birdcage coil (Robarts Research Institute, London, Canada). After acquisition of scout images, manual global shimming of unsuppressed water signal and T1-weighted imaging, a $2 \times 2 \times 2 \mathrm{~cm}$ voxel was placed on the ACC midsagittally, anterior to the genu of the corpus callosum (Figure 1). After manual shimming, a point-resolved spectroscopy sequence modified for $J$-resolved ${ }^{1} \mathrm{H}$-MRS was used. The two-dimensional (2D) $J$-resolved sequence collected 48 TE-stepped spectra from the voxel, with the TE ranging from 30 to $500 \mathrm{~ms}$ in $10-\mathrm{ms}$ increments $\quad(\mathrm{TR}=2.0 \mathrm{~s}$, acquisition bandwidth $=2 \mathrm{kHz}$, repetitions $=16$; nominal voxel volume $=8 \mathrm{~cm}^{3}$; approximate scan duration $=28 \mathrm{~min}$ ). This was repeated on a midsagittal $2 \times 2 \times 2 \mathrm{~cm}$ POC voxel (Figure 1). The total time in magnet was $75-90 \mathrm{~min}$. The water resonance full-widths at half-maximum were $9.9 \pm 1.4$ and $9.3 \pm$ $0.8 \mathrm{~Hz}$ for scan $1,10.1 \pm 1.3$ and $8.9 \pm 0.7 \mathrm{~Hz}$ for scan 2 , and $9.9 \pm 1.0$ and $9.1 \pm 0.7 \mathrm{~Hz}$ for scan 3 (in the ACC and POC, respectively; no between-scan differences).

\section{MRS Data Processing and Analysis}

For each voxel, the 48 free-induction decays (FIDs) were Gaussian filtered, zero padded, and Fourier transformed in f1 to produce $128 \mathrm{~J}$-resolved spectral FIDs over the $100-\mathrm{Hz}$ bandwidth. We used the spectral-fitting package, LCModel (version 6.0-1) (Provencher, 1993) and a serial, onedimensional (1D) spectral-fitting technique, developed in this laboratory, for the quantification of the central $50 \mathrm{~Hz}$ region $(-25$ to $+25 \mathrm{~Hz} ; 64$ spectral FIDs) of the $100 \mathrm{~Hz}$ $J$-resolved spectral data series (Figure 2). Briefly, our serial, 1D J-resolved spectral quantification procedure involves breaking down the $J$-resolved data set into separate, 1D spectral extractions. Each separate extraction is then fitted by the LCModel using a GAMMA-simulated template, in which the spectral basis set for each $J$-resolved extraction has been theoretically modeled. Once all of the $64 \mathrm{~J}$-resolved spectral extractions are fitted, the raw, metabolite integrals for each extraction over the central $50-\mathrm{Hz}$ region in $\mathrm{f} 1$ are summed up to produce a final metabolite integral. Our serial 1D quantification approach improves spectral quantification by using all available signals rather than by fitting
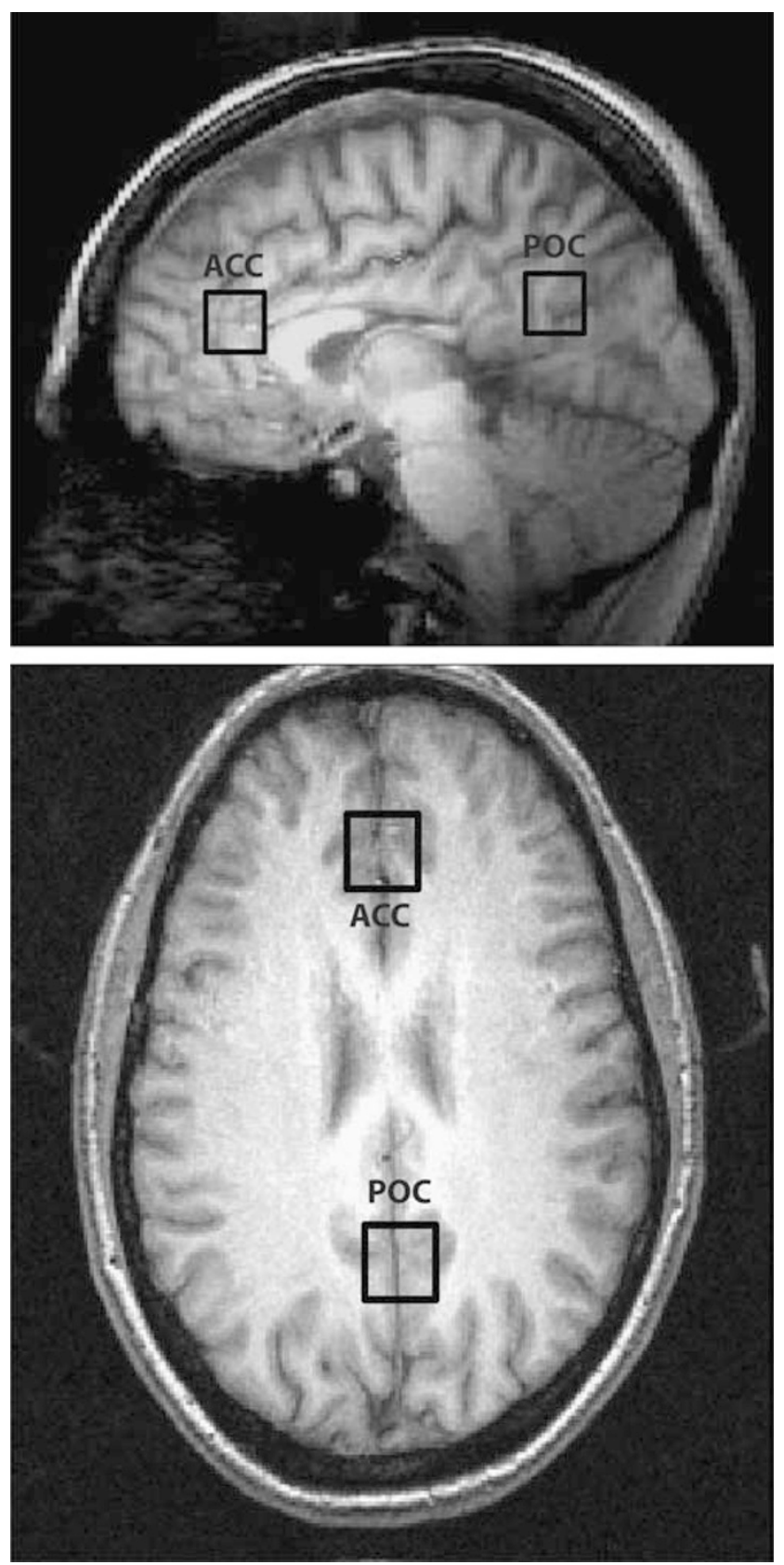

Figure I Parasagittal (top) and axial (bottom) views of the brain from $\mathrm{TI}$-weighted images illustrate the anterior cingulate cortex (ACC) and parieto-occipital cortex (POC) voxel placement in one subject.

select single $J$-resolved extractions (Jensen et al, 2009; Schulte and Boesiger, 2006a; Schulte et al, 2006b), and has been shown to greatly improve the accuracy and precision of separate measurements of the coupled and highly overlapped resonances of glutamate and glutamine. Details of our fitting procedure, including GAMMA generation of theoretical spectra for use as basis sets and fitting of individual spectral extractions, were previously reported (Öngür et al, 2008).

LCModel provides Cramer-Rao lower bounds (CRLBs), an estimate of the variance associated with fitting. Each voxel yielded 64 CRLBs for each metabolite, ranging from 
a
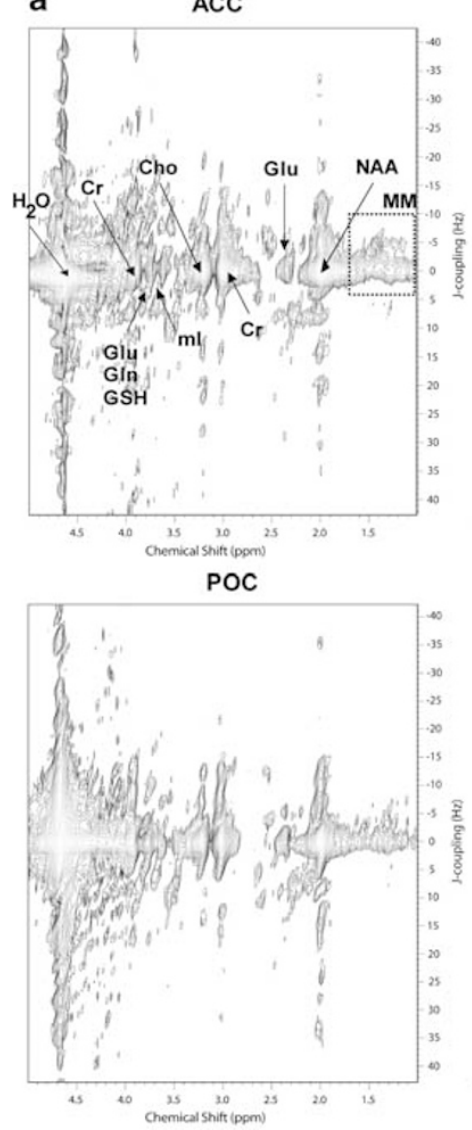

b

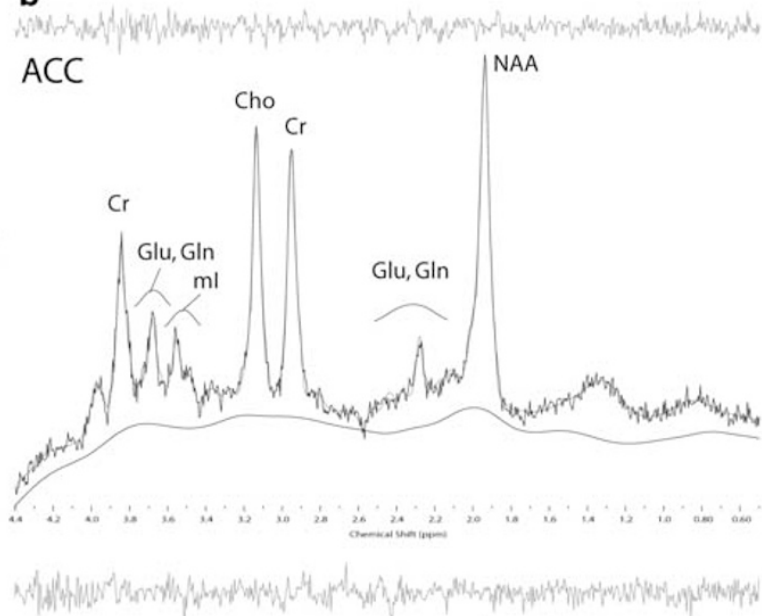

POC

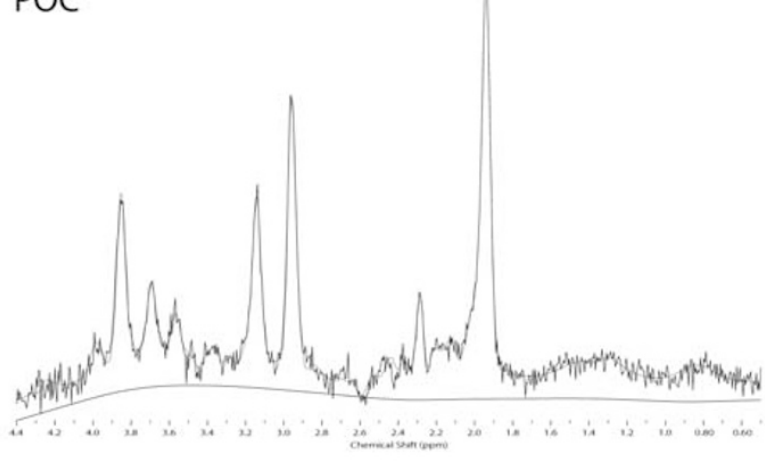

Figure 2 (a) Contour plots of real 2D spectra from the anterior cingulate cortex (ACC) and parieto-occipital cortex (POC) in one subject. In each case, the $X$ axis is frequency ( $\mathrm{f} 2$ in p.p.m.) and the $Y$ axis is J ( $\mathrm{fl}$ in $\mathrm{Hz}$ ). The spectral region from approximately -40 to $+40 \mathrm{~Hz}$ is shown. The main metabolite resonances recognizable in the plots are labeled. Although myo-inositol ( $\mathrm{ml}$ ) and glutamine (Gln) resonances are not well resolved in these plots, the additional information available from 2D MRS allows improved fitting of these metabolites. Cho, choline; Cr, creatine; Glu, glutamate, GSH, glutathione; $\mathrm{H}_{2} \mathrm{O}$, water; MMs, macromolecules; NAA, $\mathrm{N}$-acetylaspartate. (b) Sample ID spectra extracted from the same 2D data sets at $J=0.0 \mathrm{~Hz}$. Raw data are visible as a black line and the LCModel spectral fit is in red, with the residual shown in the top panel. Metabolite resonances identifiable in this spectral extraction are labeled; it must be noted that glutamine (Gln) resonances are not apparent at J=0.0 Hz. Cho, choline; Cr, creatine; Glu, glutamate; ml, myo-inositol; NAA, N-acetylaspartate. The color reproduction of this figure is available on the html full text version of the manuscript.

$<5 \%$ in regions of high certainty (close to $J=0 \mathrm{~Hz}$ ), to $>50 \%$ in regions of low certainty. To accurately reflect the reliability of the $2 \mathrm{D}$-fitting procedure, we report an average CRLB value weighted by the signal-to-noise ratio (SNR) of each spectral extraction (Table 1) on the basis of the NAA resonance. Owing to the T2-weighted signal decay with increasing TE, the signal weighting in the J-resolved spectral series is very biased, with the highest SNR being in the central $(J=0.0 \mathrm{~Hz})$ region and decreasing rapidly toward the outer, higher-frequency regions in f1. As the metabolite amplitudes from the central $J$-resolved spectral extractions with high SNR contribute more to the summed metabolite integral, we deemed it appropriate to weigh the CRLB values for each metabolite from each spectral extraction by this weighting function derived from NAA. The use of standard quadratic error propagation calculations to derive our final CRLB would be invalid as the noise in our J-resolved spectral series is highly correlated because of the Fourier transform, resulting in an underestimation of the true error. Conversely, linear addition of the CRLB values across $\mathrm{f} 1$ would unfairly bias our final error estimate by the high CRLB values from the low SNR, higher-frequency spectral extractions, resulting in overinflated error estimates. We found that our weighted average calculation best represents the test-retest metabolite variance observed in repeated measures using this $J$-resolved technique (Jensen et al, 2009), and is thus the best compromise to providing realistic CRLB error estimates for our summed metabolite values. There were no between-scan CRLB differences. As the mean group CRLBs were very good, we did not exclude data points on the basis of high CRLBs in this study. Therefore, we report higher CRLBs in some cases (eg, for creatine and choline in the POC in scan 3) as compared with the remaining data set. We report data from six metabolites reliably and consistently quantified in a previous test-retest study (Öngür et al, 2008). Our primary imaging measure, $\mathrm{Gln} / \mathrm{Glu}$ is reported as a ratio. Other metabolite levels are reported in arbitrary units (AU) to avoid introduction of bias through systematic drifts in the magnitude of the creatine resonance. 
Table I Mean ( \pm SD) Signal-to-Noise-Ratio-Weighted Cramer-Rao Lower Bounds Averages ${ }^{\text {a }}$

\begin{tabular}{|c|c|c|c|c|c|c|}
\hline & Glutamate & Glutamine & Creatine & NAA & Choline & Myo-inositol \\
\hline \multicolumn{7}{|l|}{ Baseline } \\
\hline$A C C$ & $6.1 \pm 1.7$ & $20.3 \pm 8.8$ & $2.1 \pm 0.7$ & $3.3 \pm 1.4$ & $4.2 \pm 0.9$ & $5.1 \pm 2.0$ \\
\hline $\mathrm{POC}$ & $4.9 \pm 1.1$ & $26.1 \pm 9.6$ & $1.4 \pm 0.2$ & $1.6 \pm 0.5$ & $4.4 \pm 0.9$ & $3.8 \pm 0.6$ \\
\hline \multicolumn{7}{|l|}{ Day 2} \\
\hline $\mathrm{POC}$ & $5.5 \pm 1.1$ & $28.6 \pm 12.1$ & $1.6 \pm 0.3$ & $1.7 \pm 0.3$ & $4.4 \pm 0.9$ & $4.2 \pm 0.7$ \\
\hline \multicolumn{7}{|l|}{ Week 6} \\
\hline ACC & $6.0 \pm 2.5$ & $23.5 \pm 7.3$ & $1.9 \pm 0.7$ & $2.7 \pm 1.0$ & $4.2 \pm 1.0$ & $4.5 \pm 2.1$ \\
\hline $\mathrm{POC}$ & $5.8 \pm 3.8$ & $22.6 \pm 6.1$ & $8.6 \pm 23.0$ & $2.2 \pm 2.4$ & $9.7 \pm 17.4$ & $7.8 \pm 13.1$ \\
\hline
\end{tabular}

Abbreviations: ACC, anterior cingulate cortex; NAA, N-acetylaspartate; POC, parieto-occipital cortex.

a $\vee$ alues are presented in percentage averages.

\section{Image Segmentation}

Tissue segmentation of T1-weighted images into GM, WM, and cerebrospinal fluid (CSF) was performed using FMRIB's Automated Segmentation Tool (Oxford, UK). The percentage of GM in the ACC and POC was $61 \pm 6 \%$ and $58 \pm 4 \%$, $59 \pm 9 \%$ and $57 \pm 3 \%$, and $62 \pm 8 \%$ and $60 \pm 5 \%$ for scans 1 , 2 , and 3 , respectively, whereas that of WM was $18 \pm 4 \%$ and $30 \pm 3 \%, 19 \pm 5 \%$ and $31 \pm 3 \%$, and $18 \pm 3 \%$ and $28 \pm 3 \%$ for scans 1,2 , and 3 , respectively. There were no between-scan differences in these values.

\section{Statistical Analysis}

For the primary analysis of the change in MRS measures and clinical measures over time, and the association of change in MRS measures with change in HAM-D, we used random regression models, which we refer to as our 'longitudinal analysis.' These models allow inclusion of all observed data and account for the correlation of observations from the same individual (Diggle et al, 1994; Fitzmaurice et al, 2004; Gibbons et al, 1993). For these models, we used generalized estimating equations (GEEs) to adjust SEs for the correlation of observations within individuals.

For change in clinical measures over time, the model included the clinical measure as the outcome and time (modeled as a continuous variable). The coefficient for the time term in this model quantifies the rate of clinical improvement, which we expressed as the estimated change in the measure at week 6 .

For change in MRS measures over time, our model included the MRS measure as the outcome, time (baseline, day 2 , and week 6 , modeled as unordered categories), and region (ACC or POC). We first tested for a time-by-region interaction, and if there was no significant interaction $(p<0.10)$, we tested for main effects of time. If there was a significant main effect of time $(p<0.05)$, we performed twoway comparisons for each time point against the other two.

For the association between change in MRS measures and change in HAM-D, our model included the clinical measure as the outcome, change from baseline to day 2 or week 6 in the MRS measure, time, and change-by-time interaction. The coefficient for the interaction term in this model quantifies the rate of change in HAM-D associated with change in the MRS measure. An interaction coefficient significantly different from zero indicates that change in the MRS measure is associated with change in HAM-D.

As secondary analyses for the change in clinical measures and for the association of change in MRS measures with change in HAM-D, we used change from baseline to week 6 (as measured by week 6 value minus baseline value, with missing values for week 6 imputed by last observation carried forward) as the measure of change in clinical measures; we refer to these models as our 'end point analysis.' To assess the change in clinical measures, we used a one-sample $t$-test to evaluate the null hypothesis that the value of the difference between baseline and week 6 values was equal to zero. To assess the association of change in MRS measures with change in HAM-D, we used random regression models, with GEE to account for the correlation of MRS measures from both the ACC and POC within individuals.

All analyses were performed using Stata 9.2 software. As CSF contains negligible amounts of any metabolites, and as there was variation in the amount of CSF in each voxel, we adjusted for CSF content by dividing the metabolite level by the proportion of non-CSF content, so that the levels were normalized to where they would be if there were no CSF content, based on the average metabolite level observed for a non-CSF tissue (it must be noted that no adjustment is necessary for the Gln/Glu ratio, which remains the same regardless of the proportion of CSF content). It is noteworthy that results without correction for CSF content were virtually identical to those with correction. We used Cohen's d (Cohen, 1977) as a measure of effect size. $\alpha$ was set at 0.05 , two-tailed. It must be noted that our results are presented without correction for multiple comparisons, given that the large number of correlated outcome measures makes proper adjustment for multiple comparisons difficult. Accordingly, the reader should bear in mind that some 
results, especially those of marginal significance $(0.01<p<0.05)$, may represent chance associations.

\section{RESULTS}

\section{Clinical Trial Analyses}

Participants. A total of 31 participants signed informed consent documents for the study from 6 September 2007 to 17 December 2008. Of these, 16 were withdrawn from the study before receiving treatment (medical or laboratory contraindication $(N=5)$, did not meet full criteria for $\mathrm{BD}$, currently depressed $(N=3)$, unable to tolerate MRI scan $(N=2)$, participant decision $(N=2)$, active substance dependence $(N=1)$, active psychosis $(N=1)$, positive urine drug screen $(N=1)$, active self-injurious impulses $(N=1))$. One additional participant was withdrawn during the study after discovery of a structural brain abnormality on MRI. This participant's data were not included in the final analysis. Baseline clinical features are presented in Table 2.

Of the 14 evaluable participants, 11 completed 6 weeks of treatment and 3 withdrew; 1 of these had no post-baseline clinical evaluations. Of the 13 participants with at least one post-baseline clinical visit, 11 were titrated to $150 \mathrm{mg}$ of riluzole daily, and 9 of the 11 participants continuing in the study after 3 weeks reached $200 \mathrm{mg}$ daily. Three of these participants subsequently returned to $150 \mathrm{mg}$ daily because of adverse events at $200 \mathrm{mg}$. The mean (SD) dose at end point was 181.8 (25.2) $\mathrm{mg}$ daily.

Nine participants were taking concomitant psychotropic medications as follows: (1) venlafaxine, lorazepam, and

Table 2 Participant Demographics and Clinical Information

\begin{tabular}{|c|c|}
\hline Characteristics & $N=14$ \\
\hline Age, mean (SD) & $44.5(12.0)$ \\
\hline Range & $24-60$ \\
\hline \multicolumn{2}{|l|}{ Sex } \\
\hline Female, N (\%) & $7(50)$ \\
\hline Male, N (\%) & $7(50)$ \\
\hline Caucasian non-hispanic, N (\%) & $14(100)$ \\
\hline \multicolumn{2}{|l|}{ Bipolar type } \\
\hline Type I, N (\%) & $4(29)$ \\
\hline Type 2, N (\%) & $10(7 \mid)$ \\
\hline \multicolumn{2}{|l|}{ Treatment resistance (current episode) } \\
\hline Medication naive, $N(\%)$ & $3(21)$ \\
\hline$<2$ failed medication trials, $N(\%)$ & $3(21)$ \\
\hline$\geqslant 2$ failed medication trials, $N(\%)$ & $8(58)$ \\
\hline Hamilton Depression Rating Scale, mean (SD) & $22.3(3.2)$ \\
\hline Montgomery-Asberg Depression Rating Scale, mean (SD) & $27.1(3.6)$ \\
\hline Young Mania Rating Scale, mean (SD) & $2.9(1.7)$ \\
\hline Clinical Global Impression-Severity Scale, mean (SD) & $4.5(0.5)$ \\
\hline
\end{tabular}

zolpidem, (2) quetiapine and ziprasidone, (3) bupropion, trazodone, and diazepam, (4) lorazepam, (5) ziprasidone, venlafaxine, and clonazepam, (6) fluoxetine, clonazepam, and zolpidem, (7) quetiapine, citalopram, duloxetine, and trazodone, (8) escitalopram and trazodone, and (9) omega-3 fatty acids. Two of these participants entered the study after the washout of excluded medications, such as (1) lamotrigine, amphetamine/dextroamphetamine, and acetaminophen/oxycodone and (2) amitriptyline. Five participants were taking no psychotropic medications during the study. One of these five participants electively discontinued citalopram 2 weeks before study entry.

Adverse events reported by more than one participant included nausea $(N=6)$, headache $(N=4)$, diarrhea $(N=4)$, insomnia $(N=4)$, constipation $(N=3)$, sedation $(N=3)$, neck pain $(N=3)$, dyspnea $(N=2)$, dizziness $(N=2)$, dry mouth $(N=2)$, and back pain $(N=2)$. No participant experienced a serious adverse event. There were no changes in vital signs or clinical laboratory values suggestive of drug-related toxicity.

Clinical measures. In both the random regression and end point analyses, we found a highly significant decrease, with very large effect sizes (Cohen's $d>2.0$ ), in HAM-D, MADRS, and CGI-S scores (Figure 3; Table 3); YMRS scores decreased significantly in the end point analysis, but not in the random regression analysis (Table 3 ).

\section{Metabolite Analyses}

Gln/Glu ratio. The mean metabolite levels for each time point and region are presented in Table 4. As shown in Figure 4, the mean Gln/Glu ratio increased markedly from baseline to day 2. Although the pattern of changes was more pronounced in the ACC than in the POC (Figure 4), there was no significant brain region-by-time interaction $(p=0.19)$. The mean $\mathrm{Gln} /$ Glu ratio showed a significant main effect of time $(p=0.049)$. The difference between day 2 and baseline was a large and statistically significant effect (estimated mean difference day 2 minus baseline (95\% confidence interval) $0.10(0.01,0.18) ; d=1.2 ; p=0.023)$, but the differences between week 6 and baseline and between week 6 and day 2 were not significant (mean difference week 6 minus baseline $0.05(-0.06,0.16), p=0.35$; and

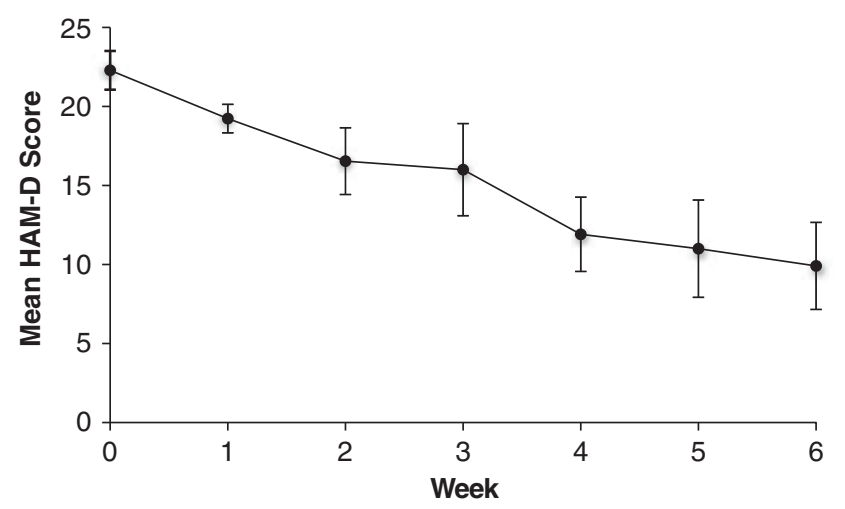

Figure 3 The mean scores on the Hamilton Depression Rating Scale (HAM-D) over 6 weeks of riluzole treatment. Error bars represent $95 \%$ confidence interval. 
Table 3 Clinical Outcome Measures Before and After 6 Weeks of Treatment with Riluzole and Analysis of Change in Outcome Measures in 14 Individuals with Bipolar Depression

\begin{tabular}{|c|c|c|c|c|c|c|c|c|}
\hline \multirow{3}{*}{ Outcome measure } & \multirow{3}{*}{$\begin{array}{l}\text { Baseline } \\
(N=14) \\
\text { Mean (SD) }\end{array}$} & \multirow{3}{*}{$\begin{array}{l}\text { Last observation } \\
\qquad \begin{array}{c}(N=\text { I3) } \\
\text { Mean (SD) }\end{array}\end{array}$} & \multicolumn{3}{|c|}{ Longitudinal analysis $^{a}$} & \multicolumn{3}{|c|}{ End point analysis ${ }^{b}$} \\
\hline & & & \multicolumn{3}{|c|}{ 6-week change } & \multicolumn{3}{|c|}{ 6-week change } \\
\hline & & & Estimate $(95 \% \mathrm{Cl})$ & $P$-value & $d^{c}$ & Estimate $(95 \% \mathrm{Cl})$ & $P$-value & $d^{c}$ \\
\hline Hamilton Depression Rating Scale & $22.2(3.2)$ & $11.0(6.6)$ & $-12.6(-16.5,-8.6)$ & $<0.001$ & 3.4 & $-12.5(-17.7,-7.2)$ & $<0.001$ & 3.0 \\
\hline Clinical Global Impression-Severity & $4.5(0.5)$ & $3.0(1.2)$ & $1.6(-2.4,-0.8)$ & $<0.001$ & 2.2 & $-1.5(-2.5,-0.6)$ & 0.004 & 2.0 \\
\hline Young Mania Rating Scale & $2.9(1.7)$ & $1.4(1.3)$ & $-0.6(-2.0,0.7)$ & 0.38 & 0.49 & $-1.5(-4.2,-0.7)$ & 0.009 & 1.8 \\
\hline
\end{tabular}

${ }^{\mathrm{a}}$ Based on all observations from all 14 participants at all visits from baseline to last observation.

based on change from baseline to last observation from 13 participants who completed at least one post-baseline evaluation.

${ }^{\circ}$ Cohen's $d$ effect size.

Table 4 Mean $\left( \pm\right.$ SD) Metabolite Levels ${ }^{a}$

\begin{tabular}{|c|c|c|c|c|c|c|c|c|}
\hline & Gln/Glu ratio & Glu $^{\mathrm{b}}$ & $G \ln ^{b}$ & Glu+Gln & $\mathrm{Cr}^{\mathrm{b}}$ & NAA & $\mathrm{Cho}^{\mathrm{b}}$ & $\mathbf{m l}^{\mathbf{b}}$ \\
\hline \multicolumn{9}{|l|}{ Baseline } \\
\hline ACC & $0.34 \pm 0.11$ & $2.13 \pm 0.67$ & $0.69 \pm 0.25$ & $2.82 \pm 0.79$ & $2.27 \pm 0.31$ & $1.71 \pm 0.40$ & $0.26 \pm 0.06$ & $1.86 \pm 0.43$ \\
\hline $\mathrm{POC}$ & $0.30 \pm 0.12$ & $1.19 \pm 0.19$ & $0.36 \pm 0.16$ & $1.55 \pm 0.30$ & $1.20 \pm 0.17$ & $1.43 \pm 0.28$ & $0.08 \pm 0.02$ & $0.92 \pm 0.16$ \\
\hline \multicolumn{9}{|l|}{ Day 2} \\
\hline \multicolumn{9}{|l|}{ Week 6} \\
\hline ACC & $0.38 \pm 0.2$ & $2.01 \pm 0.48$ & $0.69 \pm 0.30$ & $2.69 \pm 0.49$ & $2.37 \pm 0.22$ & $1.94 \pm 0.42$ & $0.25 \pm 0.05$ & $2.04 \pm 0.42$ \\
\hline $\mathrm{POC}$ & $0.36 \pm 0.26$ & $1.17 \pm 0.26$ & $0.40 \pm 0.21$ & $1.57 \pm 0.34$ & $1.11 \pm 0.38$ & $1.53 \pm 0.27$ & $0.08 \pm 0.02$ & $0.86 \pm 0.29$ \\
\hline
\end{tabular}

Abbreviations: ACC, anterior cingulate cortex; Cho, choline; Cr, creatine; Gln, glutamine; Glu, glutamate; ml, myo-inositol; NAA, N-acetylaspartate; POC, parietooccipital cortex.

a Metabolite levels are represented in arbitrary units and are adjusted for voxel cerebrospinal fluid content.

bWe did not have a priori hypotheses regarding change in these metabolites. Exploratory analysis assessing differences in levels of each metabolite over time showed no significant effect of time for any metabolite ( $P \geqslant 0.25$ for all comparisons).

mean difference week 6 minus week $2-0.04(-0.12,0.03)$, $p=0.25$, respectively). There were no significant differences between time points across the study in the total pool of glutamate plus glutamine ( $p=0.68$ for main effect of time), glutamate $(p=0.25)$, or glutamine $(p=0.44)$.

$\mathrm{N}$-acetylaspartate. NAA showed little change from baseline to day 2, but increased from baseline to week 6 (Figure 5). The mean NAA level showed a significant effect of time $(p=0.001)$. There was no significant brain region-by-time interaction $(p=0.48)$, but the pattern of findings was more pronounced in the ACC (Figure 5). The difference between week 6 and baseline was a large effect $(d=1.2)$ that was statistically significant (mean difference week 6 minus baseline $1.9(0.01,3.6) \mathrm{AU} ; p=0.035)$, as was the difference between week 6 and day 2 (mean difference week 6 minus day $22.4(1.1,3.8) \mathrm{AU} ; d=1.9 ; p<0.001)$, but the difference between day 2 and baseline was not significant (mean difference day 2 minus baseline $-0.5(-2.4,1.3) \mathrm{AU}$; $p=0.57$ ).

Association of clinical response with change in metabolites. We found no significant association between clinical response (as measured by change from baseline to week 6 in HAM-D) and change from baseline to week 2 or from baseline to week 6 in the Gln/Glu ratio with clinical response, using either longitudinal or end point analyses (Table 5).

The association between change in NAA and baseline to week 6 change in HAM-D showed a significant brain regionby-NAA interaction from baseline to day $2(p=0.010)$ and from baseline to week $6(p<0.001)$ for the longitudinal 
analysis. Therefore, we analyzed associations separately by region (Table 5). For change in NAA from baseline to day 2, we found no significant association with change in HAM-D in the ACC, but a very strong negative association $(d=9.6)$ with clinical improvement in the POC. For change in NAA from baseline to week 6 , we found a strong positive association with clinical improvement $(d=1.4)$ in the ACC, approaching statistical significance (Figure 6), and again a strong negative association $(d=2.3)$ in the POC (Figure 7).

We also carried out an a posteriori analysis of the association between the change in NAA at week 6 and the

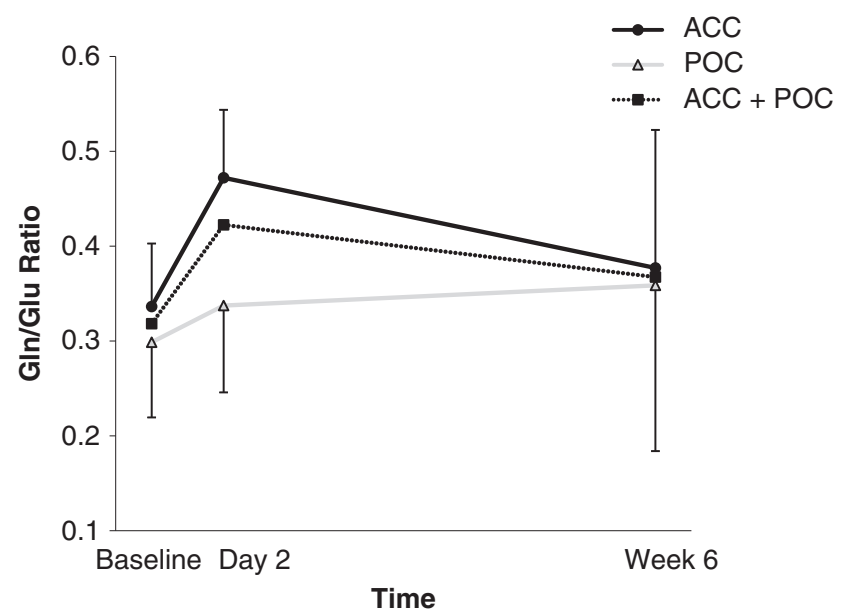

Figure 4 The glutamine/glutamate (Gln/Glu) ratio in the anterior cingulate cortex (ACC) and parieto-occipital cortex (POC) as measured by J-resolved proton magnetic resonance spectroscopy ('H-MRS) at baseline, day 2 of riluzole treatment, and week 6 of riluzole treatment. Dashed line shows the mean Gln/Glu ratio in combined ACC and POC as measured by 'H-MRS at baseline, day 2 of riluzole treatment, and week 6 of riluzole treatment. Error bars represent $95 \%$ confidence interval. change in the Gln/Glu ratio at day 2, using random linear regression, with the former measure as outcome and the latter as predictor. We found a significant brain region-bychange in Gln/Glu ratio interaction $(p=0.006)$, and consequently used linear regression to assess the association within each region. In the ACC, we found a strong $(d=1.6)$ association approaching statistical significance (coefficient $-22.4(-53.0,4.2) ; p=0.082)$, but no significant association in the POC (coefficient $-2.1(-6.6,2.3)$; $p=0.30)$.

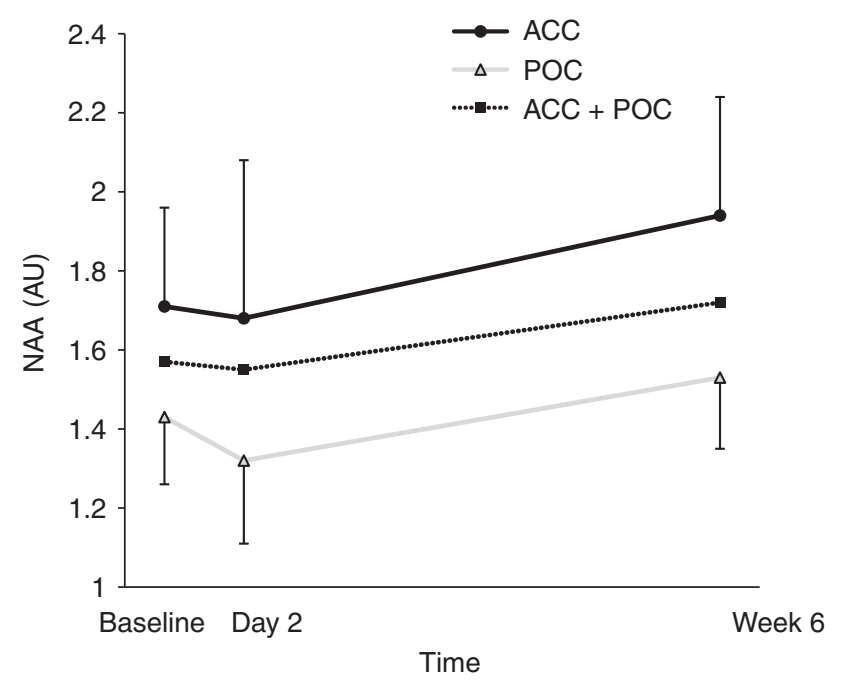

Figure 5 The $\mathrm{N}$-acetylaspartate (NAA) level in the anterior cingulate cortex (ACC) and parieto-occipital cortex (POC) as measured by J-resolved proton magnetic resonance spectroscopy ( $\mathrm{H}$-MRS) at baseline, day 2 of riluzole treatment, and week 6 of riluzole treatment. Dashed line shows mean NAA level in combined ACC and POC as measured by 'H-MRS at baseline, day 2 of riluzole treatment, and week 6 of riluzole treatment. NAA levels are represented in arbitrary units (AU). Error bars represent $95 \%$ confidence interval.

Table 5 Association Between Change in Hamilton Depression Rating Scale (HAM-D) Scores ${ }^{a}$ and Change in MRS measures (Glutamine/ Glutamate Ratio and NAA)

\begin{tabular}{|c|c|c|c|c|c|c|}
\hline \multirow{2}{*}{ MRS measure } & \multicolumn{3}{|c|}{ Longitudinal analysis } & \multicolumn{3}{|c|}{ End point analysis } \\
\hline & Estimate $^{a}(95 \% \mathrm{Cl})$ & $P$-value & d & Estimate $^{a}(95 \% \mathrm{Cl})$ & $P$-value & d \\
\hline Baseline to day 2 change & $3.7(-7.1,14.5)$ & 0.50 & 0.36 & $7.5(-4.6,19.6)$ & 0.22 & 0.68 \\
\hline Baseline to week 6 change & $3.5(-6.2,13.2)$ & 0.48 & 0.42 & $7.7(-4.3,19.6)$ & 0.25 & 0.58 \\
\hline \multicolumn{7}{|l|}{ ACC } \\
\hline Baseline to day 2 change & $-1.1(-5.4,3.3)$ & 0.64 & 0.30 & $-0.7(-9.0,7.6)$ & 0.86 & 0.12 \\
\hline Baseline to week 6 change & $-4.0(-8.0,0.0)$ & 0.053 & 1.4 & $-6.0(16.7,4.7)$ & 0.23 & 0.93 \\
\hline \multicolumn{7}{|l|}{$\mathrm{POC}$} \\
\hline Baseline to day 2 change & $31.4(26.2,36.7)$ & $<0.001$ & 9.6 & $38.2(-5.3,81.7)$ & 0.073 & 1.8 \\
\hline
\end{tabular}

${ }^{a}$ Estimate represents mean increase in the difference in HAM-D score from baseline to week 6 (week 6 minus baseline) for each increase of I in the difference in MRS measure (either glutamine/glutamate ratio or N-acetylaspartate (NAA) level (in arbitrary units)) from baseline to either day 2 or week 6 (day 2 or week 6 minus baseline).

${ }^{\mathrm{b}}$ Results reported separately for each region because of significant interaction effect of region on association. 


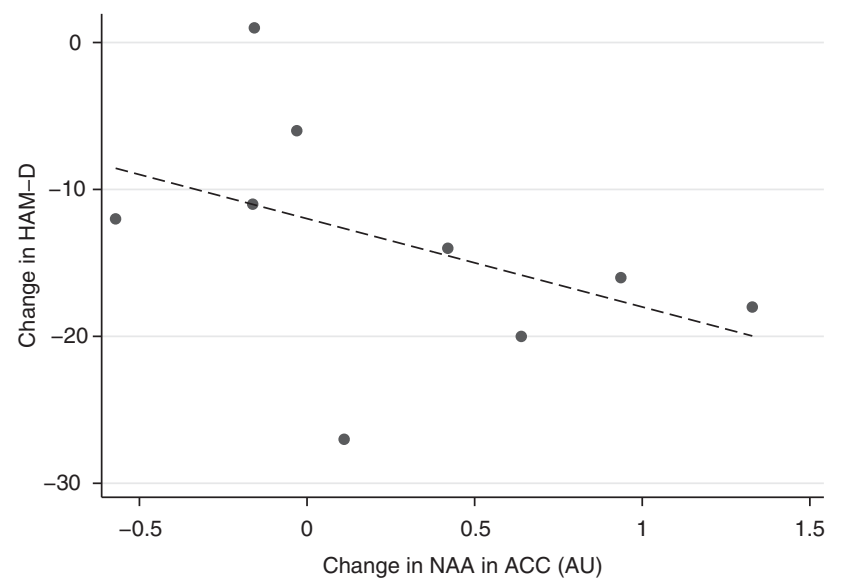

Figure 6 Scatter plot for change in N-acetylaspartate (NAA) in the anterior cingulate cortex (ACC) vs change in Hamilton Depression Rating Scale (HAM-D) from baseline to week 6 of riluzole treatment. Change in NAA is represented in arbitrary units (AU). It must be noted that this figure has one less data point than does Figure 7 because of technically unevaluable data in the ACC for one subject.

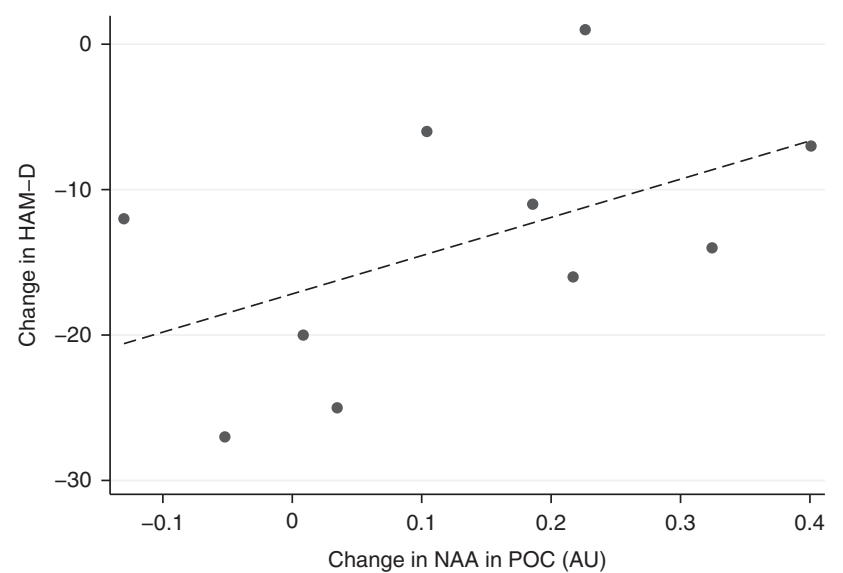

Figure 7 Scatter plot for change in N-acetylaspartate (NAA) in the parieto-occipital cortex (POC) vs change in Hamilton Depression Rating Scale (HAM-D) from baseline to week 6 of riluzole treatment. Change in NAA is represented in arbitrary units $(A U)$.

Finally, to further explore the stability of our findings, we conducted additional statistical analyses (1) restricted to study completers $(N=11),(2)$ comparing those who were taking concomitant psychotropic medications $(N=9)$ with those who were not $(N=5)$, and (3) comparing those with treatment resistance (defined as two or more failed medication trials during the current episode $(N=8)$ ) with those who had failed only one medication trial or those who were medication naive $(N=6)$. These exercises produced generally similar estimates of the magnitude of effects to those of the analyses reported above.

\section{DISCUSSION}

In 14 bipolar-depressed participants, riluzole produced (1) a rapid and pronounced increase in the Gln/Glu ratio by day
2 , followed by a decline by week $6,(2)$ an increase in NAA levels at week 6 , and (3) a significant reduction in depression symptoms, which was positively associated with increase in NAA from baseline to week 6 in the ACC, but negatively associated in the POC.

\section{Riluzole and Glutamatergic Neurotransmission}

The marked increase in the Gln/Glu ratio at day 2 of riluzole treatment was unexpected, given previous in vitro findings that riluzole decreases presynaptic glutamate release (Doble et al, 1992; Martin et al, 1993; Prakriya and Mennerick, 2000; Wang et al, 2004). However, animal studies provide some support for our finding. For example, studies in rats treated chronically with riluzole have shown increased glutamate-glutamine cycling, hypothesized to result primarily from increased oxidative metabolism through the TCA cycle both in neurons (Chowdhury et al, 2008) and in glia (Banasr et al, 2008; Valentine and Sanacora, 2009). Interestingly, this result is consistent with other studies (Kalra et al, 2006; Mu et al, 2000), suggesting that riluzole may have mitochondrial-enhancing properties, in addition to its previously suggested glutamate-modulating effects.

Strikingly, riluzole significantly increased Gln/Glu ratios by day 2, before antidepressant response, but this change seemed to have largely dissipated by week 6 , when the clinical response had emerged (although it should be noted that the decline from day 2 to week 6 did not reach statistical significance). Possibly, the early impact of riluzole on glutamate-glutamine cycling increased the Gln/Glu ratio, which then returned to baseline levels by week 6 after a new steady-state level was reached. Interestingly, the total pool (glutamate plus glutamine) was stable over the course of the study; the day 2 increase in the Gln/Glu ratio reflected a simultaneous increase in glutamine and decrease in glutamate, with both metabolites returning to their approximate baseline levels, along with the Gln/Glu ratio, at week 6 . This reciprocal relationship between glutamate and glutamine seems to be consistent with a shift in the glutamate-glutamine cycle after riluzole treatment. Collectively, the above-mentioned findings suggest that riluzole acutely upregulates glutamate-glutamine cycling in vivo. It is also intriguing that this pattern was more pronounced in the ACC (which is implicated in processing emotional stimuli) than in the POC, although this pattern did not reach statistical significance.

\section{Riluzole and NAA}

Our finding of increased NAA after 6 weeks of riluzole treatment is consistent with a previous finding of increased hippocampal NAA in patients with generalized anxiety disorder who showed a clinical response after 8 weeks of riluzole treatment (Mathew et al, 2008) - although one cannot be certain that the mechanisms of NAA increase were similar in both cases. Although not completely understood, reduced NAA levels are widely interpreted to indicate reduced neuronal health, either through neuronal/ axonal loss or through compromised neuronal metabolism (Moffett et al, 2007). In contrast to our finding of no acute change in NAA after treatment, a previous study in patients with ALS has shown an increase in NAA after only 1 day of 
riluzole treatment (Kalra et al, 2006), which the authors theorized was a result of rapid metabolic enhancement. However, there are several potential reasons for the discrepancy between the two studies, most notably differences in the disorders (ALS vs BD) and regions of interest (ACC and POC vs motor cortex) studied. Importantly, previous studies have indicated that the turnover rate of NAA is in the range of 2-3 days (Choi and Gruetter, 2004; Moffett et al, 2007), suggesting that day 2 of treatment may be too early to observe significant increases in NAA resulting from rapid enhancement of mitochondrial function. Therefore, although not reflected in NAA measurements at day 2, it is plausible that riluzole treatment acutely results in metabolic enhancement and increased glutamateglutamine cycling which chronically leads to neural plasticity and symptom improvement, as suggested by others (Mathew et al, 2008). The significant association between increase in the ACC Gln/Glu ratio from baseline to day 2 and increase in NAA at week 6 seems to support this hypothesis. Clearly, further studies examining the impact of riluzole treatment on mitochondrial functioning are required to explain these findings more thoroughly.

However, there are alternative interpretations of a larger NAA resonance. For example, prolongation in NAA transverse (T2) relaxation time could lead to larger NAA resonance without changes in metabolite levels. T2 relaxation times reflect the molecular microenvironment and can be modulated by cell size, cell membrane turnover, and brain blood volume. Notably, abnormalities in T2 relaxation time have been identified in bipolar patients (Öngür et al, $2009 \mathrm{~b}$ ), and brain water T2 relaxation time changes have been reported in patients with $\mathrm{BD}$ after treatment with omega-3 fatty acids (Hirashima et al, 2004). Therefore, further work is required to determine whether riluzole treatment induces changes in $\mathrm{T} 2$ relaxation times.

\section{Association with Clinical Response}

The positive association between clinical response and increase in NAA in the ACC at week 6 suggests that improvement in depression is associated with enhanced neuronal integrity and function in the ACC. This finding is consistent with the results of the study by Mathew et al (2008), and provides additional evidence that NAA might represent a surrogate end point for clinical trials of plasticity-enhancing agents, such as riluzole, in psychiatric illnesses. Given the significant association between increase in the ACC Gln/Glu ratio from baseline to day 2 and increase in NAA at week 6, one might also expect that the former measure would predict later clinical response. However, this was not borne out in our statistical analysis-possibly as a result of small sample size or incomplete imaging data sets as a result of subject attrition and technically unevaluable imaging data. Thus, we might have failed to detect medium or even large effects (as reflected by our estimates of effect sizes from 0.36 to 0.68 in our two analyses) - warranting further exploration of the predictive value of the Gln/Glu ratio in larger similar studies.

Surprisingly, clinical response was negatively associated with NAA in the POC. It is possible that the POC may harbor biological abnormalities distinct from the ACC and perhaps from other brain regions, resulting in different effects on glutamatergic activity after riluzole treatment. However, it will be necessary to collect additional data with a larger sample to replicate these findings, and if replicated, to more fully characterize these differences.

Overall, our findings augment the growing evidence that glutamatergic abnormalities contribute to the neurobiology of BD. Lacking a comparison group with $\mathrm{BD}$, we cannot confirm decreased Gln/Glu ratios at baseline-but our findings suggest that riluzole may reset a pathologically depressed level of synaptic glutamatergic activity, possibly through the rapid enhancement of glutamate-glutamine cycling. Interestingly, this increase seems temporary, with return to baseline by 6 weeks (although this decline from day 2 to week 6 did not reach statistical significance as mentioned previously), perhaps after a new steady-state glutamate-glutamine cycling rate has been achieved or through autoregulatory processes that intervene after a new synaptic strength 'set point' is established.

Possibly early riluzole-induced increases in glutamateglutamine cycling amplify postsynaptic $\alpha$-amino-3-hydroxy5-methyl-4-isoxazole propionic acid (AMPA) receptor levels, leading to increased AMPA/N-methyl- $D$-aspartate (NMDA) receptor stimulation, previously hypothesized as a convergent mechanism for antidepressant action (Schloesser et al, 2008; Zarate et al, 2006a). Increased AMPA receptor 'throughput' may ultimately cause downstream neuroproliferative effects through the induction of brain-derived neurotrophic factor and multiple intracellular signaling cascades (Lauterborn et al, 2000; Lauterborn et al, 2009; Mackowiak et al, 2002; Voss et al, 2007; Wu et al, 2004; Zhang et al, 2009). These speculations accord with previous evidence (Schloesser et al, 2008). For example, riluzole enhances the surface expression of GluR1 and GluR2 AMPA receptor subtypes in cultured hippocampal neurons after only 3 days of treatment ( $\mathrm{Du}$ et al, 2007). Similarly, the potent NMDA receptor antagonist ketamine elicits a similar increase in AMPA/NMDA neurotransmission associated with a rapid and prolonged antidepressant effect in animal studies (Maeng et al, 2008). Moreover, ketamine infusion increases ACC glutamine levels in healthy individuals, possibly reflecting enhanced glutamatergic neurotransmission (Rowland et al, 2005). However, although there are potential parallels between the antidepressant mechanisms of riluzole and ketamine, there are likely differences in the time to onset of these effects. Ketamine is reported to cause clear antidepressant effects within $2 \mathrm{~h}$ of administration (Zarate et al, 2006b). We did not measure mood symptoms at day 2 of this study, and were therefore unable to assess for potential rapid antidepressant effects after riluzole treatment. However, previous studies have shown a longer time to onset of antidepressant effects with riluzole ranging from 1 to 5 weeks of treatment (Sanacora et al, 2007; Zarate et al, 2004, 2005). Notably, lamotrigine, which typically takes several weeks to achieve antidepressant effects, shows similar effects on AMPA receptor expression in vitro as riluzole and ketamine (Du et al, 2007). Therefore, although increased AMPA/NMDA neurotransmission may be a common mechanism of antidepressant action shared by all three of these medications, ketamine's rapidity of action may be derived from a unique pharmacological characteristic, such as potency of effect. 
Although the primary mechanism of action of riluzole is believed to be presynaptic inhibition of glutamate release, it may also increase glutamate reuptake through the enhancement of glial excitatory amino-acid transporter functioning (Fumagalli et al, 2008; Valentine and Sanacora, 2009). Thus, riluzole may rapidly enhance glutamate-glutamine cycling, while increasing clearance of glutamate from the synapse. These complementary effects increase synaptic glutamatergic activity while moderating the intensity of NMDA receptor stimulation and limiting overflow of glutamate into the extrasynaptic space where it can stimulate extrasynaptic NMDA receptors-both hypothesized mechanisms of excitotoxicity (Hardingham, 2006).

\section{Limitations}

Several methodological limitations to this study should be considered. First, the small sample size limits statistical power. Second, our open-label design leaves open the possibility of a placebo effect, making it difficult to distinguish biological effects of riluzole from nonspecific effects of clinical improvement. Third, although we excluded patients using medications that have strong evidence of glutamate-modulating properties such as lamotrigine and lithium, we included several patients using atypical antipsychotics, benzodiazepines, and selective serotonin reuptake inhibitors. Although these latter agents have not been shown to strongly impact glutamatergic neurotransmission, such effects cannot be excluded. Given the ethical and logistical problems posed by recruiting unmedicated bipolar-depressed patients and the exploratory nature of this study, we believed that our approach limited the potential contribution of concomitant medications while still allowing the enrollment of a larger population of bipolar-depressed patients. Clearly, future studies should ultimately attempt to obtain either unmedicated patients or a sample large enough to examine the impact of concomitant medications. Fourth, we did not assess the menstrual cycle status in female participants, although this factor has been shown to impact glutamatergic activity (Batra et al, 2008). Fifth, we report metabolite levels in AU. We avoided internal referencing to creatine because of recent reports suggesting creatine abnormalities in BD (Frye et al, 2007; Öngür et al, 2009a) and internal referencing to water was not possible, lacking usable water unsuppressed spectra in this study. Our approach does not correct for inter-subject sources of variance, but the longitudinal within-subject design of our study and statistical analysis mitigates the problems of variability inherent to many cross-sectional MRS studies. Sixth, the $J$-resolved ${ }^{1} \mathrm{H}$-MRS technique used in this study allows reliable quantification of multiple important metabolites, but not that of $\gamma$-aminobutyric acid (GABA), the inhibitory counterpart to the excitatory glutamate system. As glutamate and glutamine are intimately involved in GABAergic neurotransmission, measures of glutamate and glutamine are not necessarily only related to glutamatergic neurotransmission. Therefore, GABA levels would be desirable to more fully characterize riluzole's mechanism of action. Finally, it is worth noting that $J$-resolved ${ }^{1} \mathrm{H}$-MRS is not unique in its ability to resolve glutamate and glutamine, and others have used conventional (1D) MRS to quantify these metabolites at high magnetic field ( $\mathrm{Oz}$ et al, 2006; Theberge et al, 2002). As a result, the advantages of $J$-resolved ${ }^{1} \mathrm{H}$ MRS (its ability to collect additional data on difficult-toquantify metabolites (Schulte et al, 2006b) and metabolite T2 relaxation times) and its disadvantages (longer scan times and potential drift in data quality during the scan) must be weighed when designing future studies.

\section{Summary}

In bipolar-depressed patients, riluzole increased $\mathrm{Gln} / \mathrm{Glu}$ ratios at day 2, and by week 6 reduced depressive symptoms and increased NAA - perhaps reflecting enhanced neuronal plasticity. Further studies using $J$-resolved ${ }^{1} \mathrm{H}$-MRS should pursue the possibility that Gln/Glu ratios represent an early biomarker of response to therapies that enhance neuronal plasticity.

\section{ACKNOWLEDGEMENTS}

This study was supported by a grant from Jim and Pat Poitras (DÖ and BPB) and by Grants 1K23MH079982-02 (DÖ) and R01MH058618 (PFR) from the National Institute of Mental Health. BPB was supported by the Clinical Investigator Training Program (CITP) through the Beth Israel Deaconess Medical Center-Harvard/MIT Division of Health Sciences and Technology in collaboration with Pfizer Inc. and Merck and Co. and by the Sidney R Baer, Jr, Foundation through a NARSAD Young Investigator Award. We would like to acknowledge Sanofi-aventis for providing riluzole tablets for this study (Note: Sanofi-aventis provided no additional financial support for this study and had no input into the design or analysis of this study).

\section{DISCLOSURE}

BPB has received research grant support from Eli Lilly. JIH has received research grant support from Eli Lilly, OrthoMcNeil Janssen Scientific Affairs, and Forest Laboratories, and has been a consultant for Eli Lilly and Pfizer. HGP has received research grant support from Solvay Pharmaceuticals. PFR has received research grant support from GlaxoSmithKline and Roche, and has been a consultant to Novartis, Roche, and Kyowa Hakko. None of the other authors reported any biomedical financial interests or potential conflicts of interest.

\section{REFERENCES}

Banasr M, Chowdhury GM, Terwilliger R, Newton SS, Duman RS, Behar KL et al (2008). Glial pathology in an animal model of depression: reversal of stress-induced cellular, metabolic and behavioral deficits by the glutamate-modulating drug riluzole. Mol Psychiatry (e-pub ahead of print 30 September).

Batra NA, Seres-Mailo J, Hanstock C, Seres P, Khudabux J, Bellavance $\mathrm{F}$ et al (2008). Proton magnetic resonance spectroscopy measurement of brain glutamate levels in premenstrual dysphoric disorder. Biol Psychiatry 63: 1178-1184.

Bhagwagar Z, Wylezinska M, Jezzard P, Evans J, Ashworth F, Sule A et al (2007). Reduction in occipital cortex gamma-aminobutyric acid concentrations in medication-free recovered unipolar depressed and bipolar subjects. Biol Psychiatry 61: 806-812. 
Cecil KM, DelBello MP, Morey R, Strakowski SM (2002). Frontal lobe differences in bipolar disorder as determined by proton $\mathrm{MR}$ spectroscopy. Bipolar Disord 4: 357-365.

Cheramy A, Barbeito L, Godeheu G, Glowinski J (1992). Riluzole inhibits the release of glutamate in the caudate nucleus of the cat in vivo. Neurosci Lett 147: 209-212.

Choi IY, Gruetter R (2004). Dynamic or inert metabolism? Turnover of $\mathrm{N}$-acetyl aspartate and glutathione from $\mathrm{D}$-[113C]glucose in the rat brain in vivo. $J$ Neurochem 91: 778-787.

Chowdhury GM, Banasr M, de Graaf RA, Rothman DL, Behar KL, Sanacora G (2008). Chronic riluzole treatment increases glucose metabolism in rat prefrontal cortex and hippocampus. J Cereb Blood Flow Metab 28: 1892-1897.

Cohen J (1977). Statistical Power Analysis for the Behavioral Sciences, revised edition. Academic Press: New York.

Dager SR, Friedman SD, Parow A, Demopulos C, Stoll AL, Lyoo IK et al (2004). Brain metabolic alterations in medication-free patients with bipolar disorder. Arch Gen Psychiatry 61: 450-458.

Diggle PJ, Liang KY, Zeger SL (1994). Analysis of Longitudinal Data. Oxford University Press: Oxford, England.

Doble A (1996). The pharmacology and mechanism of action of riluzole. Neurology 47(6 Suppl 4): S233-S241.

Doble A, Hubert JP, Blanchard JC (1992). Pertussis toxin pretreatment abolishes the inhibitory effect of riluzole and carbachol on $\mathrm{D}-[3 \mathrm{H}]$ aspartate release from cultured cerebellar granule cells. Neurosci Lett 140: 251-254.

Du J, Suzuki K, Wei Y, Wang Y, Blumenthal R, Chen Z et al (2007). The anticonvulsants lamotrigine, riluzole, and valproate differentially regulate AMPA receptor membrane localization: relationship to clinical effects in mood disorders. Neuropsychopharmacology 32: 793-802.

Fitzmaurice GM, Laird NM, Ware JH (2004). Applied Longitudinal Analysis. John Wiley \& Sons: Hoboken, New Jersey.

Frye MA, Watzl J, Banakar S, O’Neill J, Mintz J, Davanzo P et al (2007). Increased anterior cingulate/medial prefrontal cortical glutamate and creatine in bipolar depression. Neuropsychopharmacology 32: 2490-2499.

Fumagalli E, Funicello M, Rauen T, Gobbi M, Mennini T (2008). Riluzole enhances the activity of glutamate transporters GLAST, GLT1 and EAAC1. Eur J Pharmacol 578: 171-176.

Gibbons RD, Hedeker D, Elkin I, Waternaux C, Kraemer HC, Greenhouse JB et al (1993). Some conceptual and statistical issues in analysis of longitudinal psychiatric data. Application to the NIMH treatment of Depression Collaborative Research Program dataset. Arch Gen Psychiatry 50: 739-750.

Hardingham GE (2006). Pro-survival signalling from the NMDA receptor. Biochem Soc Trans 34(Pt 5): 936-938.

Hirashima F, Parow AM, Stoll AL, Demopulos CM, Damico KE, Rohan ML et al (2004). Omega-3 fatty acid treatment and T(2) whole brain relaxation times in bipolar disorder. Am J Psychiatry 161: 1922-1924.

Igarashi H, Kwee IL, Nakada T, Katayama Y, Terashi A (2001). 1H magnetic resonance spectroscopic imaging of permanent focal cerebral ischemia in rat: longitudinal metabolic changes in ischemic core and rim. Brain Res 907: 208-221.

Iltis I, Koski DM, Eberly LE, Nelson CD, Deelchand DK, Valette J et al (2009). Neurochemical changes in the rat prefrontal cortex following acute phencyclidine treatment: an in vivo localized (1)H MRS study. NMR Biomed 22: 737-744.

Jensen JE, Licata SC, Öngür D, Friedman SD, Prescot AP, Henry ME et al (2009). Quantification of J-resolved proton spectra in two-dimensions with LCModel using GAMMA-simulated basis sets at 4 Tesla. NMR Biomed 22: 762-769.

Kalra S, Cashman NR, Genge A, Arnold DL (1998). Recovery of $\mathrm{N}$-acetylaspartate in corticomotor neurons of patients with ALS after riluzole therapy. Neuroreport 9: 1757-1761.

Kalra S, Tai P, Genge A, Arnold DL (2006). Rapid improvement in cortical neuronal integrity in amyotrophic lateral sclerosis detected by proton magnetic resonance spectroscopic imaging. J Neurol 253: 1060-1063.

Kugaya A, Sanacora G (2005). Beyond monoamines: glutamatergic function in mood disorders. CNS Spectr 10: 808-819.

Lauterborn JC, Lynch G, Vanderklish P, Arai A, Gall CM (2000). Positive modulation of AMPA receptors increases neurotrophin expression by hippocampal and cortical neurons. J Neurosci 20: 8-21.

Lauterborn JC, Pineda E, Chen LY, Ramirez EA, Lynch G, Gall CM (2009). Ampakines cause sustained increases in brain-derived neurotrophic factor signaling at excitatory synapses without changes in AMPA receptor subunit expression. Neuroscience 159: 283-295.

Mackowiak M, O’Neill MJ, Hicks CA, Bleakman D, Skolnick P (2002). An AMPA receptor potentiator modulates hippocampal expression of BDNF: an in vivo study. Neuropharmacology 43: $1-10$

Maeng S, Zarate Jr CA, Du J, Schloesser RJ, McCammon J, Chen $G$ et al (2008). Cellular mechanisms underlying the antidepressant effects of ketamine: role of alpha-amino-3hydroxy-5-methylisoxazole-4-propionic acid receptors. Biol Psychiatry 63: 349-352.

Martin D, Thompson MA, Nadler JV (1993). The neuroprotective agent riluzole inhibits release of glutamate and aspartate from slices of hippocampal area CA1. Eur J Pharmacol 250: 473-476.

Mathew SJ, Price RB, Mao X, Smith EL, Coplan JD, Charney DS et al (2008). Hippocampal N-acetylaspartate concentration and response to riluzole in generalized anxiety disorder. Biol Psychiatry 63: 891-898.

Michael N, Erfurth A, Ohrmann P, Gossling M, Arolt V, Heindel W et al (2003). Acute mania is accompanied by elevated glutamate/ glutamine levels within the left dorsolateral prefrontal cortex. Psychopharmacology (Berl) 168: 344-346.

Michael N, Erfurth A, Pfleiderer B (2009). Elevated metabolites within dorsolateral prefrontal cortex in rapid cycling bipolar disorder. Psychiatry Res 172: 78-81.

Mlynarik V, Kohler I, Gambarota G, Vaslin A, Clarke PG, Gruetter $\mathrm{R}$ (2008). Quantitative proton spectroscopic imaging of the neurochemical profile in rat brain with microliter resolution at ultra-short echo times. Magn Reson Med 59: 52-58.

Moffett JR, Ross B, Arun P, Madhavarao CN, Namboodiri AM (2007). N-Acetylaspartate in the CNS: from neurodiagnostics to neurobiology. Prog Neurobiol 81: 89-131.

$\mathrm{Mu}$ X, Azbill RD, Springer JE (2000). Riluzole improves measures of oxidative stress following traumatic spinal cord injury. Brain Res 870: 66-72.

Öngür D, Jensen JE, Prescot AP, Stork C, Lundy M, Cohen BM et al (2008). Abnormal glutamatergic neurotransmission and neuronal-glial interactions in acute mania. Biol Psychiatry 64: 718-726.

Öngür D, Prescot AP, Jensen JE, Cohen BM, Renshaw PF (2009a). Creatine abnormalities in schizophrenia and bipolar disorder. Psychiatry Res 172: 44-48.

Öngür D, Prescot AP, Jensen JE, Rouse ED, Cohen BM, Renshaw $\mathrm{PF}$ et al (2009b). T2 relaxation time abnormalities in bipolar disorder and schizophrenia. Magn Reson Med (in press).

Oz G, Terpstra M, Tkac I, Aia P, Lowary J, Tuite PJ et al (2006). Proton MRS of the unilateral substantia nigra in the human brain at 4 Tesla: detection of high GABA concentrations. Magn Reson Med 55: 296-301.

Phillips ML, Drevets WC, Rauch SL, Lane R (2003). Neurobiology of emotion perception II: implications for major psychiatric disorders. Biol Psychiatry 54: 515-528.

Post RM (2005). The impact of bipolar depression. J Clin Psychiatry 66(Suppl 5): 5-10.

Prakriya M, Mennerick S (2000). Selective depression of lowrelease probability excitatory synapses by sodium channel blockers. Neuron 26: 671-682. 
Provencher SW (1993). Estimation of metabolite concentrations from localized in vivo proton NMR spectra. Magn Reson Med 30: 672-679.

Rothman DL, Behar KL, Hyder F, Shulman RG (2003). In vivo NMR studies of the glutamate neurotransmitter flux and neuroenergetics: implications for brain function. Annu Rev Physiol 65: 401-427.

Rowland LM, Bustillo JR, Mullins PG, Jung RE, Lenroot R, Landgraf $\mathrm{E}$ et al (2005). Effects of ketamine on anterior cingulate glutamate metabolism in healthy humans: a 4 -T proton MRS study. Am J Psychiatry 162: 394-396.

Sanacora G, Gueorguieva R, Epperson CN, Wu YT, Appel M, Rothman DL et al (2004). Subtype-specific alterations of gammaaminobutyric acid and glutamate in patients with major depression. Arch Gen Psychiatry 61: 705-713.

Sanacora G, Kendell SF, Levin Y, Simen AA, Fenton LR, Coric V et al (2007). Preliminary evidence of riluzole efficacy in antidepressant-treated patients with residual depressive symptoms. Biol Psychiatry 61: 822-825.

Sanacora G, Rothman DL, Mason G, Krystal JH (2003). Clinical studies implementing glutamate neurotransmission in mood disorders. Ann N Y Acad Sci 1003: 292-308.

Sanacora G, Zarate CA, Krystal JH, Manji HK (2008). Targeting the glutamatergic system to develop novel, improved therapeutics for mood disorders. Nat Rev Drug Discov 7: 426-437.

Schloesser RJ, Huang J, Klein PS, Manji HK (2008). Cellular plasticity cascades in the pathophysiology and treatment of bipolar disorder. Neuropsychopharmacology 33: 110-133.

Schulte RF, Boesiger P (2006a). ProFit: two-dimensional prior-knowledge fitting of J-resolved spectra. NMR Biomed 19: 255-263.

Schulte RF, Lange T, Beck J, Meier D, Boesiger P (2006b). Improved two-dimensional J-resolved spectroscopy. NMR Biomed 19: 264-270.

Theberge J, Al-Semaan Y, Williamson PC, Menon RS, Neufeld RW, Rajakumar $\mathrm{N}$ et al (2003). Glutamate and glutamine in the anterior cingulate and thalamus of medicated patients with chronic schizophrenia and healthy comparison subjects measured with 4.0-T proton MRS. Am J Psychiatry 160: 2231-2233.
Theberge J, Bartha R, Drost DJ, Menon RS, Malla A, Takhar J et al (2002). Glutamate and glutamine measured with $4.0 \mathrm{~T}$ proton MRS in never-treated patients with schizophrenia and healthy volunteers. Am J Psychiatry 159: 1944-1946.

Valentine GW, Sanacora G (2009). Targeting glial physiology and glutamate cycling in the treatment of depression. Biochem Pharmacol (e-pub ahead of print 17 April).

Voss OP, Milne S, Sharkey J, O’Neill MJ, McCulloch J (2007). Molecular mechanisms of neurite growth with AMPA receptor potentiation. Neuropharmacology 52: 590-597.

Wang SJ, Wang KY, Wang WC (2004). Mechanisms underlying the riluzole inhibition of glutamate release from rat cerebral cortex nerve terminals (synaptosomes). Neuroscience 125: 191-201.

Wu X, Zhu D, Jiang X, Okagaki P, Mearow K, Zhu G et al (2004). AMPA protects cultured neurons against glutamate excitotoxicity through a phosphatidylinositol 3-kinase-dependent activation in extracellular signal-regulated kinase to upregulate BDNF gene expression. J Neurochem 90: 807-818.

Zarate Jr CA, Payne JL, Quiroz J, Sporn J, Denicoff KK, Luckenbaugh D et al (2004). An open-label trial of riluzole in patients with treatment-resistant major depression. $A m \mathrm{~J}$ Psychiatry 161: 171-174.

Zarate Jr CA, Quiroz JA, Singh JB, Denicoff KD, De Jesus G, Luckenbaugh DA et al (2005). An open-label trial of the glutamate-modulating agent riluzole in combination with lithium for the treatment of bipolar depression. Biol Psychiatry 57: $430-432$.

Zarate Jr CA, Singh J, Manji HK (2006a). Cellular plasticity cascades: targets for the development of novel therapeutics for bipolar disorder. Biol Psychiatry 59: 1006-1020.

Zarate Jr CA, Singh JB, Carlson PJ, Brutsche NE, Ameli R, Luckenbaugh DA et al (2006b). A randomized trial of an $\mathrm{N}$ methyl-D-aspartate antagonist in treatment-resistant major depression. Arch Gen Psychiatry 63: 856-864.

Zhang QG, Han D, Hu SQ, Li C, Yu CZ, Wang $\mathrm{R}$ et al (2009). Positive modulation of AMPA receptors prevents downregulation of GluR2 expression and activates the Lyn-ERK1/2CREB signaling in rat brain ischemia. Hippocampus (e-pub ahead of print 27 March 2009). 